\title{
Bulge properties and dark matter content of early-type barred galaxies
}

\author{
E. M. Corsini \\ Dipartimento di Astronomia, Università di Padova, vicolo dell'Osservatorio 3, \\ I-35122 Padova, Italy \\ email: enricomaria.corsini@unipd.it
}

\begin{abstract}
The dynamics of a barred galaxy depends on the pattern speed of its bar. The only direct method for measuring the pattern speed of a bar is the Tremaine-Weinberg technique. This method is best suited to the analysis of the distribution and dynamics of the stellar component. Therefore it has been mostly used for early-type barred galaxies. Most of them host a classical bulge. On the other hand, a variety of indirect methods, which are based on the analysis of the distribution and dynamics of the gaseous component, has been used to measure the bar pattern speed in late-type barred galaxies. Nearly all the measured bars are as rapidly rotating as they can be. By comparing this result with high-resolution numerical simulations of bars in dark matter halos, it is possible to conclude that these bars reside in maximal disks.
\end{abstract}

Keywords. galaxies: kinematics and dynamics, galaxies: structure

\section{Introduction}

More than $60 \%$ of all bright nearby galaxies are disk-dominated systems. Bars are seen in about $60 \%$ of them. This is also true at higher redshift. In fact, the bar fraction is constant out to $z \sim 1$ (see Marinova \& Jogee 2006 and references therein). Therefore bars are a common feature in the central regions of disk galaxies. Their growth is partly regulated by the exchange of angular momentum with the stellar disk and the dark matter (DM) halo. For this reason the dynamical evolution of bars can be used to constrain the content and distribution of DM in the inner regions of galaxy disks.

The morphology and dynamics of a barred galaxy depend on the pattern speed of the bar, $\Omega_{B}$. Usually, it is parametrized with the bar rotation rate $\mathcal{R} \equiv D_{L} / a_{B}$. This is the distance-independent ratio between the corotation radius, $D_{L}=V_{\mathrm{c}} / \Omega_{B}$ (where $V_{\mathrm{c}}$ is the disk circular velocity), and the length of bar semi-major axis, $a_{B}$. At $D_{L}$ the gravitational and centrifugal forces cancel out in the rest frame of the bar. As far as the value of $\mathcal{R}$ concerns, if $\mathcal{R}<1.0$ the stellar orbits are elongated perpendicular to the bar and the bar dissolves. For this reason, self-consistent bars cannot exist in this regime. Bars with $\mathcal{R} \gtrsim 1.0$ are close to rotate as fast they can, and there is not a priori reason for $\mathcal{R}$ to be significantly larger than 1.0. The knowledge of $\mathcal{R}$ allows to distinguish between fast bars $(1.0 \leqslant \mathcal{R} \leqslant 1.4)$ and slow bars $(\mathcal{R}>1.4)$. This choice does not imply anything about the actual rotation velocity of bar.

\section{Measuring the bar pattern speed}

A variety of indirect methods has been used to measure $\Omega_{B}$ and corresponding $\mathcal{R}$. They are based on the identification of morphological features with the location of Lindblad's resonances, comparison of the observed gas velocity and density fields with numerical models of gas flows, and analysis of the offset and shape of dust lanes which traces the location shocks in the gas flows. All these methods are model dependent and are mostly 
used for late-type barred galaxies because they rely on the presence of gas. Nearly all the measured bars are found to be fast (see Elmegreen 1996 for a review).

A model-independent method for measuring $\Omega_{B}$ is the Tremaine-Weinberg method (Tremaine \& Weinberg 1984, TW). They showed that if a tracer satisfies the continuity equation, then $\Omega_{B}$ is determined from two observationally accessible quantities: the luminosity-weighted mean of positions $\mathcal{X}$ and the luminosity-weighted mean of line-ofsight velocities $\mathcal{V}$ of the tracer. It is

$$
\mathcal{X} \Omega_{B} \sin i=\mathcal{V}
$$

with

$$
\mathcal{X}=\frac{\int_{-\infty}^{+\infty} X \Sigma(X, Y) d X}{\int_{-\infty}^{+\infty} \Sigma(X, Y) d X} \quad \text { and } \quad \mathcal{V}=\frac{\int_{-\infty}^{+\infty} V_{\operatorname{los}}(X, Y) \Sigma(X, Y) d X}{\int_{-\infty}^{+\infty} \Sigma(X, Y) d X}
$$

where $X$ and $Y$ are the Cartesian coordinates chosen to be aligned with the observed major and minor axis of the galaxy disk. The position $X$, velocity $V_{\text {los }}$, and surface brightness $\Sigma$ of the tracer are measured along a strip which is parallel to the disk major axis and offset by $Y$. This strip actually corresponds to the aperture of either a slit or a pseudo-slit in long-slit and integral-field spectroscopy, respectively. Plotting $\mathcal{V}$ versus $\mathcal{X}$ for the different strips produces a straight line with slope $\Omega_{B} \sin i$. The inclination $i$ of the galaxy disk is derived from the analysis of the surface brightness distribution.

The assumption underlying the TW method is that the observed surface brightness is proportional to the surface density of the tracer, as for old stellar populations in the absence of significant and patchy obscuration of dust. Thus, the method has been applied largely to absorption-line spectra of early-type barred galaxies (Table 1). All the objects listed in Table 1 are bright galaxies, except for NGC 4431. Corsini et al. (2007) found that the probability that the bar of this dwarf galaxy ends close to its corotation radius (i.e., it is fast) is about twice as likely as that the bar is much shorter than corotation radius (i.e., it is slow). A fast bar in NGC 4431 would suggest a common formation mechanism of the bar both in bright and dwarf galaxies. If their disks were previously stabilized by massive DM halos, bars were not produced by tidal interactions because they would be slowly rotating (Noguchi 1999). But, this is not the case even for galaxies which show signs of weak tidal interaction with a close companion (e.g., NGC 4431 and NGC 1023). This finding implies there is no difference between TW measurements of the stellar component in isolated or mildly interacting barred galaxies. All the measured bars are consistent with being in fast. This is particularly true when galaxies with small uncertainties on $\mathcal{R}$ (e.g., $\Delta \mathcal{R} / \mathcal{R}<0.3$ ) are considered.

Most of the studied SB0 bulges have the photometric and kinematic properties of classical bulges (Aguerri et al. 2005). In particular, they follow the Faber-Jackson correlation, lie on the fundamental plane and those for which stellar kinematics are available rotate as fast as the bulges of unbarred galaxies. The remaining bulges have disk properties typical of pseudobulges.

A successful application of the TW method to the stellar component of late-type barred galaxies will remedy the selection bias present in the current sample of directly measured pattern speeds. This problem could be addressed by using near-infrared spectroscopy in order to deal with dust obscuration. In fact, according to Gerssen \& Debattista (2007) realistic values of dust attenuation change the observed $\Omega_{B}$ by less than 30 per cent.

Extensions of the TW method to multiple pattern speeds were proposed by Corsini et al. (2003), Maciejewski (2006), and Merrifield et al. (2006). They can be applied to measure pattern speeds of nested bars, as in NGC 2950. The primary bar of this 
Table 1. Barred galaxies with $\Omega_{B}$ measured by applying TW method to the stellar component

\begin{tabular}{|c|c|c|c|c|c|c|c|}
\hline $\begin{array}{c}\text { Galaxy } \\
\text { (1) }\end{array}$ & $\begin{array}{c}\text { Morp. Type } \\
(2)\end{array}$ & $\begin{array}{c}D \\
(\mathrm{Mpc}) \\
(3)\end{array}$ & $\begin{array}{c}a_{B} \\
(\operatorname{arcsec}) \\
(4)\end{array}$ & $\begin{array}{c}\Omega_{B} \\
\left(\mathrm{~km} \mathrm{~s}^{-1} \underset{(5)}{\operatorname{arcsec}}-1\right)\end{array}$ & $\begin{array}{c}D_{L} \\
(\operatorname{arcsec}) \\
(6)\end{array}$ & $\begin{array}{l}\mathcal{R} \\
(7)\end{array}$ & $\begin{array}{l}\text { Ref. } \\
(8)\end{array}$ \\
\hline ESO $139-G 09$ & $(\mathrm{R}) \mathrm{SAB0}^{0}(\mathrm{rs})$ & 71.9 & $17_{-3}^{+6}$ & $21.4 \pm 5.8$ & $15_{-3}^{+5}$ & $0.8_{-0.2}^{+0.3}$ & $A+03$ \\
\hline ESO 281-G31 & $\mathrm{SB}^{+}(\mathrm{rs})$ & 45.2 & $11 \pm 1$ & $10.5 \pm 4.1$ & $20_{-4}^{+12}$ & $1.8_{-0.4}^{+1.2}$ & $\mathrm{G}+03$ \\
\hline IC 874 & $\mathrm{SB} 0^{0}(\mathrm{rs})$ & 34.7 & $20 \pm 5$ & $7.0 \pm 2.4$ & $27_{-7}^{+13}$ & $1.4_{-0.4}^{+0.4}$ & $\mathrm{~A}+03$ \\
\hline NGC 271 & $\left(\mathrm{R}^{\prime}\right) \mathrm{SBab}(\mathrm{rs})$ & 50.3 & $29 \pm 1$ & $7.8 \pm 4.3$ & $44_{-16}^{+30}$ & $1.5_{-0.5}^{+1.40}$ & $\mathrm{G}+03$ \\
\hline NGC 936 & $\mathrm{SB}^{+}(\mathrm{rs})$ & 14.9 & 50 & $4.8 \pm 1.1$ & $69 \pm 15$ & $1.4_{-0.4}^{+0.5}$ & MK95 \\
\hline NGC 1023 & $\mathrm{SB}^{-}(\mathrm{rs})$ & 5.8 & $69 \pm 5$ & $5.1 \pm 1.8$ & $53_{-14}^{+29}$ & $0.8_{-0.3}^{+0.4}$ & $\mathrm{D}+02$ \\
\hline NGC 1308 & $\mathrm{SB} 0 / \mathrm{a}(\mathrm{r})$ & 82.4 & $12_{-3}^{+2}$ & $39.7 \pm 13.9$ & $9_{-2}^{+5}$ & $0.8_{-0.2}^{+0.4}$ & $\mathrm{~A}+03$ \\
\hline NGC 1358 & $\mathrm{SAB} 0 / \mathrm{a}(\mathrm{r})$ & 51.6 & $19 \pm 3$ & $9.3 \pm 4.5$ & $23_{-7}^{+19}$ & $1.2_{-0.4}^{+1.2}$ & $\mathrm{G}+03$ \\
\hline NGC 1440 & $\left(\mathrm{R}^{\prime}\right) \mathrm{SB}^{0}(\mathrm{rs})$ : & 18.4 & $24_{-5}^{+6}$ & $7.4 \pm 1.7$ & $38_{-7}^{+71}$ & $1.6_{-0.3}^{+0.4}$ & $\mathrm{~A}+03$ \\
\hline NGC 2503 & $\operatorname{SBbc}(r)$ & 46.0 & 34 & $6.6 \pm 1.6$ & 45 & $1.4 \pm 0.3$ & $\mathrm{~T}+07$ \\
\hline NGC 2950 & $(\mathrm{R}) \mathrm{SB}^{0}(\mathrm{r})$ & 19.7 & $34 \pm 2$ & $11.2 \pm 2.4$ & $32_{-6}^{+9}$ & $1.0_{-0}^{+0.3}$ & $\mathrm{C}+03$ \\
\hline NGC 3412 & $\mathrm{SB}^{0}(\mathrm{~s})$ & 16.0 & $31 \pm 3$ & $4.4 \pm 1.2$ & $47_{-10}^{+16}$ & $1.5_{-0.3}^{+0.2}$ & $\mathrm{~A}+03$ \\
\hline NGC 3992 & $\mathrm{SBbc}(\mathrm{rs})$ & 16.4 & $57 \pm 12$ & $5.7 \pm 0.4$ & $45 \pm 3$ & $0.8 \pm 0.2$ & $\mathrm{G}+03$ \\
\hline NGC 4245 & $\mathrm{SB} 0 / \mathrm{a}(\mathrm{r}):$ & 15.6 & 38 & $4.7 \pm 1.9$ & 43 & $1.1 \pm 0.5$ & $\mathrm{~T}+07$ \\
\hline NGC 4431 & $\mathrm{dSB} 0 / \mathrm{a}$ & 15.0 & $22 \pm 2$ & $7.4 \pm 1.8$ & $13_{-3}^{+4}$ & $0.6_{-0.1}^{+0.2}$ & $\mathrm{C}+07$ \\
\hline NGC 4596 & $\mathrm{SB}^{+}(\mathrm{r})$ & 29.3 & 53 & $3.9 \pm 1.0$ & $60_{-12}^{+20}$ & $1.2_{-0.2}^{+0.1}$ & $\mathrm{G}+99$ \\
\hline NGC 7079 & $\mathrm{SB}^{0}(\mathrm{~s})$ & 34.0 & $25 \pm 4$ & $8.4 \pm 0.2$ & $31 \pm 1$ & $1.2_{-0.2}^{+0.3}$ & DW04 \\
\hline
\end{tabular}

NOTE - Col.(2): Morphological classification from RC3, except for ESO 281-G31 (NED) and NGC 4431 (Barazza et al. 2002). NGC 2950 is a double-barred galaxy and the listed values refer to its primary bar. Col.(3): Distance obtained as $V_{\mathrm{CBR}} / H_{0}$ with $V_{\mathrm{CBR}}$ from RC3 and $H_{0}=75 \mathrm{~km} \mathrm{~s}^{-1} \mathrm{Mpc}^{-1}$. Col.(4): Bar length. Col.(5): Bar pattern speed. Col.(6): Bar corotation radius. Col.(7): Bar rotation rate. Col.(8): Reference papers.

double-barred galaxy has a smaller pattern speed than the secondary one, which is possibly counterrotating (Corsini et al. 2003; Maciejewski 2006).

In most cases the presence of shocks, conversion of gas between different phases, and star formation on short timescales prevent the application of the TW method to gas. These limitations were explored by Rand \& Wallin (2004) and Hernández et al. (2005) with numerical experiments. Ionized (Hernandez et 2004, 2005; Emsellem et al. 2006; Fathi et al. 2007), atomic (Bureau et al. 1999), and molecular gas (Zimmer et al. 2004; Rand \& Wallin 2004) were used to derive $\Omega_{B}$ in a number of barred galaxies.

\section{Dark matter distribution in barred galaxies}

Using perturbation theory Weinberg (1985) predicted that a bar would lose angular momentum to a massive DM halo through dynamical friction, slowing down in the process. This prediction was confirmed in N-body simulations (Debattista \& Sellwood 1998, 2000; Athanassoula 2003; O'Neill \& Dubinski 2003). They found that bars are slowed efficiently within a few rotation periods if a substantial density of DM is present in the region of the bar. On the other hand, if the mass distribution is dominated by the stellar disk throughout the inner few disk scale-lengths, then the bar remains fast for a long time. These findings were challenged by Valenzuela \& Klypin (2003) who claimed that strong bars can rotate rapidly $(\mathcal{R}=1.3-1.7)$ in centrally-concentrated DM halos for long periods. This anomalous behaviour was attributed by Sellwood \& Debattista (2006) to the adopted numerical technique which suppresses the decreasing density of particles with angular momentum about the principal resonances usually responsible for friction. 
This phenomenon could arise in nature due, for example, to gas flows in the bar. But this is a metastable state and mild perturbations quickly restore the frictional drag. Thus the accurate measurement of $\Omega_{B}$ provides a way to discriminate whether the central regions of the host galaxy are dominated by baryons or by DM.

Nearly all the measured bars are found to be consistent with fast rotators. Direct measurements of bar pattern speeds use stars as the tracer population in SB0's and early-type spirals and gas in later Hubble types. On the other hand, the model-dependent techniques rely on the presence of gas and were mostly applied to late-type spirals. Fast bars require that the disk, in which they formed, contributes most of the rotational support in the inner parts of the galaxy. This means that barred galaxies have maximal disks. This conclusion holds also for bright unbarred galaxies. The comparison of the Tully-Fisher relations for unbarred and barred galaxies shows they have a comparable fraction of DM at a given radius (Debattista \& Sellwood 2000; Courteau et al. 2003).

\section{References}

Aguerri, J. A. L., Debattista, V. P., \& Corsini, E. M. 2003, MNRAS, 338, 465 (A+03)

Aguerri, J. A. L., et al. 2005, A\&BA, 434, 109

Athanassoula, E. 2003, MNRAS, 341, 1179

Barazza, F. D., Binggeli, B., \& Jerjen, H. 2002, A\&A, 391, 823

Bureau, M., et al. 1999, AJ, 118, 2158

Corsini, E. M., Debattista, V. P., \& Aguerri, J. A. L. 2003, ApJ, 599, L29 (C+03)

Corsini, E. M., et al. 2007, ApJ, 659, L121 (C+07)

Courteau, S., et al. 2003, ApJ, 594, 208

Debattista, V. P., Corsini, E. M., \& Aguerri, J. A. L. 2002, MNRAS, 332, 65 (D+02)

Debattista, V. P. \& Sellwood, J. A. 1998, ApJ, 493, L5

Debattista, V. P. \& Sellwood, J. A. 2000, ApJ, 543, 704

Debattista, V. P. \& Williams, T. B. 2004, ApJ, 605, 714 (DW04)

Elmegreen, B. 1996, in Barred Galaxies, ed. R. Buta et al., ASP Conf. Ser., (San Francisco: ASP), vol. 91, p. 197

Emsellem, E., et al. 2006, MNRAS, 365, 367

Fathi, K., et al. 2007, ApJ, in press (arXiv:0708.1081)

Gerssen, J., \& Debattista, V. P. 2007, MNRAS, 378, 189

Gerssen, J., Kuijken, K., \& Merrifield, M. R. 1999, MNRAS, 306, 926 (G+99)

Gerssen, J., Kuijken, K., \& Merrifield, M. R. 2003, MNRAS, 345, 261 (G+03)

Hernández, O., et al. 2004, in Penetrating Bars Through Masks of Cosmic Dust, ed. D. L. Block et al., ASSL (Dordrecht: Kluwer), vol. 319, p. 781

Hernández, O., et al. 2005, ApJ, 632, 253

Maciejewski, W. 2006, MNRAS, 371, 451

Marinova, I. \& Jogee, S. 2007, ApJ, 659, 1176

Merrifield, M. R. \& Kuijken, K. 1995, MNRAS, 274, 933 (MK95)

Merrifield, M. R., Rand, R. J., \& Meidt, S. E. 2006, MNRAS, 366, L17

Noguchi, M. 1999, ApJ, 514, 77

O'Neill, J. K. \& Dubinski, J. 2003, MNRAS, 346, 251

Rand, R. J. \& Wallin, J. F. 2004, ApJ, 614, 142

Sellwood, J. A. \& Debattista, V. P. 2006, ApJ, 639, 868

Tremaine, S. \& Weinberg, M. D. 1984, ApJ, 282, L5

Treuthardt, P., et al. 2007, AJ, 134, $1195(\mathrm{~T}+07)$

Valenzuela, O. \& Klypin, A. 2003, MNRAS, 345, 406

Weinberg, M. D. 1985, MNRAS, 213, 451

Westpfahl, D. J. 1998, ApJS, 115, 203

Zimmer, P., Rand, R. J., \& McGraw, J. T. 2004, ApJ, 607, 285 